\title{
Diversidad genética de ibias (Oxalis tuberosa Molina) y cubios (Tropaeolum tuberosum Ruíz y Pavón) en Boyacá
}

\section{Genetic diversity of ibias (Oxalis tuberosa Molina) and cubios (Tropaeolum tuberosum Ruíz y Pavón) in Boyacá}

\author{
Ana C. Morillo ${ }^{1 *}$, Yacenia Morillo², María F. Leguizamo ${ }^{3}$ \\ Recibido para publicación: Febrero 21 de 2016 - Aceptado para publicación: Mayo 25 de 2016
}

\begin{abstract}
RESUMEN
La región andina es considerada un área que alberga una gran diversidad de especies entre ellas las raíces y tubérculos andinos como las ibias (Oxalis tuberosa Molina) y los cubios (Tropaeolum tuberosum Ruíz y Pavón), los cuales constituyen un componente básico de la dieta de las comunidades rurales. Sus tubérculos presentan altos contenidos de metabolitos primarios y secundarios que les confiere propiedades antibióticas, antioxidantes insecticidas, nematicidas, anticancerígenas y diuréticas. Teniendo en cuenta su enorme potencial y que en Boyacá no existen estudios de diversidad genética en estas especies se planteó la caracterización molecular de 10 materiales de ibias y 11 de cubios procedentes de los municipios de Soracá, Ventaquemada, Tuta, San Pedro de Iguaque y el Puente de Boyacá. El análisis mediante el coeficiente de NeiLi diferenció a la población en dos grandes grupos de acuerdo a las características morfológicas del tubérculo y a la especie. La heterocigosidad promedio estimada fue de 0,41 y un coeficiente de diferenciación genética de 0,15 . Los resultados obtenidos en este estudio mostraron la existencia de una variabilidad genética a nivel intraespecífico y flujo genético entre las dos especies evaluadas lo cual puede ser aprovechado en esquemas de mejoramiento tendientes a la producción de híbridos y la explotación de los efectos heteróticos.
\end{abstract}

Palabras clave: Tubérculos andinos, microsatélites, variabilidad, hibridación.

\begin{abstract}
The Andean region is considered as an area which has a great diversity of species including roots and Andean tubers like ibias (Oxalis tuberosa Molina) and cubios (Tropaeolum tuberosum Ruíz and Pavón). They are basic components of the diet of rural communities. Their tubers feature high content of primary and secondary metabolites that confers antibiotic, antioxidant, insecticides, nematicides, anticarcinogenic and diuretic properties. Considering their huge potential and the fact that there are no studies of genetic diversity of these species in Boyacá, the molecular characterization of 10 ibias and 11 cubios from Soracá, Ventaquemada, Tuta, San Pedro de Iguaque and Puente de Boyacá municipalities was proposed. The analysis by the coefficient of Nei-Li discriminated the population into two groups according to the morphological characteristics of the tuber and per species. The average heterozygosity estimated was 0,41 and a genetic differentiation coefficient of 0.15 . The results obtained in this study demonstrated the existence of a genetic variation at an intraspecific level and a gene flow between the two evaluated species, which can be used in breeding programs tending to the production of hybrids and the exploitation of heterotic effects.
\end{abstract}

Key words: Andean tubers, microsatellites, variability, hybridization.

\footnotetext{
1* I.A, PhD. Fitomejoramiento. Profesor Asociado UPTC, Programa Ingeniería Agronómica, Universidad Pedagógica y Tecnológica de Colombia. Tunja. Colombia. Avenida Central Norte 39-115, Tunja, Boyacá, email: ana.morillo@uptc.edu.co.

2 I.A, PhD. Fitomejoramiento. Profesor Universidad de Caldas. yacenia.morillo@ucaldas.edu.co, Universidad de Caldas, Programa Ingeniería Agronómica Calle 65 № 26-10 Manizales-Caldas.

${ }^{3}$ Ing. Agrónomo Universidad Pedagógica y Tecnológica de Colombia, Tunja, Colombia. Avenida Central Norte 39-115, Tunja, Boyacá.
} 


\section{INTRODUCCIÓN}

La región de los Andes es considerada uno de los 25 megacentros de diversidad biológica y uno de los centros de origen de Vavilov, por lo cual alberga una gran variedad de especies entre ellas las raíces y los tubérculos andinos como las ibias (O. tuberosa), el ulluco (Ullucus tuberosus Caldas), los cubios (T. tuberosum Ruíz y Pavón) y la papa (Solanum tuberosum spp. andígena) los cuales hacen parte de la dieta básica de las comunidades indígenas andinas (Malice et al. 2010).

Las ibias pertenecen a la clase Dicotyledonae, orden Geraniales, familia Oxalidaceae y al género Oxalis, es probablemente después de la papa, el tubérculo andino más conocido. Es originario de altiplano Peruano-Boliviano, cultivada en Venezuela, Perú, Bolivia, Chile, Argentina y Colombia como también en México y Nueva Zelanda en donde es apreciada por presentar colores y sabores muy particulares. Las plantas de $O$. tuberosa son rastreras, pueden llegar a medir un metro de altura, con tallos suculentos, sus hojas son trifoliadas y alternas de color verde brillante, inflorescencias pequeñas con cuatro o cinco flores, su polinización es cruzada, su fruto es una cápsula que contiene dos o tres semillas y con una gran diversidad en la forma (cilíndrica, ovoide o claviforme) y el color de los tubérculos (blancos, anaranjados, rojos, violetas, casi negros), presenta tolerancia a algunos estreses bióticos y abióticos, por lo tanto tiene una mayor adaptación a ambientes en donde otros cultivos no pueden sobrevivir (Rosero 2010). Sus tubérculos son ricos en carbohidratos, minerales, ácidos orgánicos los cuales le permiten su uso industrial, alimenticio y medicinal (Bradbury and Emshwiller 2011; Moscoe et al. 2016).

Los cubios (T. tuberosum) pertenecen a la familia Tropaeolaceae y al género Tropaeolum, el cual incluye alrededor de 86 especies distríbuidas en toda Sur América, desde
Colombia hasta el norte de Argentina, entre los 2400 hasta los 4300 msnm y también se cultiva en algunas regiones de Nueva Zelanda y Canadá (Chirinos et al. 2007), generalmente en asocio con otros tubérculos, en pequeñas áreas en donde hace parte de la seguridad alimentaria de miles de familias en los Andes (Manrique et al. 2013). Es una planta herbácea, anual, de aproximadamente $80 \mathrm{~cm}$ de altura, con diversidad en el color del follaje (varía de amarillo-verde a verde oscuro) y el tubérculo, sus flores son profusas y producen semillas viables con alta tasa de germinación (Leguizamo 2015). Es una planta que presenta una gran adaptación a condiciones extremas; tolerante a nematodos, insectos plaga; sus tubérculos tienen elevados contenidos de proteína, carbohidratos, fibra, ácido ascórbico (Vitamina C), calorías y glusinolatos, componentes que le confieren propiedades antibióticas, antioxidantes insecticidas, nematicidas, anticancerígenas y diuréticas (Chirinos et al. 2008 a, b; Manrique et al. 2013).

Las raíces y tubérculos andinos, son consideradas especies marginales, propias de comunidades rurales, con laboriosos procesos de cocción y bajo retorno económico (Jiménez et al. 2015). En los Andes colombianos (Nariño, Cauca, Cundinamarca y Boyacá), estos cultivos son herencia de los antiguos Paeces, Muiscas y Laches entre otros y se usan básicamente para el autoconsumo. En Boyacá las condiciones agroclimatológicas han permitido el desarrollo de variedades con diferentes propiedades impulsadas por el mercado del trueque y los diferentes usos alimenticios y medicinales (Rosero 2010).

Entre los tubérculos andinos existe una gran variabilidad en formas, tamaños, colores, calidades y cantidades de metabolitos primarios (almidones, minerales, proteínas, vitaminas, ácidos grasos, glucósidos, azúcares) y secundarios (saponinas, alcaloides, taninos, oxalatos, carotenos, antocianinas, betacianinas) 
(Manrique et al. 2013), los cuales deben ser tenidos en cuenta para su conservación y caracterización. Dentro de este contexto a nivel internacional países como Ecuador, Bolivia y Perú han generado investigación sobre la conservación, diversidad genética y usos alternativos de estos cultivos (Manrique et al. 2013). En Colombia, la Universidad Nacional cuenta con una colección de 65 accesiones de cubios la cual ha sido caracterizada fenotípica, bioquímica y genotípicamente (Piñeros y Ñústez 2004; Arias e Higuera 2011). En Boyacá se han adelantado procesos de investigación en conservación de ibias, cubios y rubas, en pequeños sistemas productivos de Turmequé, Ventaquemada (Rivera 2010; Aguirre et al. 2012).

La variabilidad genética de estos cultivos ha sido descrita utilizando inicialmente marcadores morfológicos y agronómicos (Rosero 2010) bioquímicos (Monteros 1997) y moleculares (Malice et al. 2010; Moscoe et al. 2016). Entre los marcadores microsatélites, los RAMs (Microsatélites Amplificados al Azar) son muy útiles para medir la diversidad genética en plantas, animales y microorganismos, además permiten detectar la variación a nivel intra e inter específico, no necesita información previa y es una metodología útil para la determinación de parámetros genéticos (Morillo et al. 2015).

Algunos de estos estudios muestran que por ser cultivos propagados vegetativamente y en peligro de extinción; entre las estrategias para conservar e incrementar su diversidad genética, en búsqueda de materiales más atractivos para el mercado, se plantea la hibridación como un mecanismo que favorece la presencia de nuevas variantes alélicas además que es común en el origen de especies poliploides del género Oxalis y Tropaeolum (Emshwiller et al. 2009; Hershkovitz et al. 2006). La hibridación y la poliploidización son dos fuerzas evolutivas importantes en las plantas como fuentes de variación de variación genética y epigenética
(Marfil et al. 2006; Rapp y Wendel 2005) en cruces interespecíficos e intergenéricos; sin embargo, la mayor limitación se presenta por las barreras de cruzabilidad entre las especies, la cual actualmente está siendo superada por el uso de herramientas biotecnológicas (Wang et al. 2013; Wen et al. 2016). Teniendo en cuenta lo anterior el objetivo de esta investigación fue estudiar la diversidad genética de las especies Oxalis tuberosa M y Tropaeolum tuberosum Ruíz y Pavón del departamento de Boyacá, mediante marcadores microsatélites RAMs con el fin de determinar las distancias genéticas y en futuro proponer estrategias de hibridación intergenéricas para generar materiales genéticos que respondan a las necesidades del agricultor, productor y comercializador.

\section{MATERIALES Y MÉTODOS}

\section{Material vegetal:}

Se colectaron hojas jóvenes de 10 materiales de ibias y 11 de cubios procedentes del departamento de Boyacá, municipios de Soracá, Ventaquemada, Tuta, San Pedro de Iguaque y el Puente de Boyacá (Tabla 1).

\section{Caracterización molecular:}

Se llevó a cabo en los laboratorios de investigación en Biología Molecular de la Universidad Pedagógica y Tecnológica de Colombia, Tunja. La extracción del ADN se hizo con el protocolo de Dellaporta et al. (1983) y se visualizó en geles de agarosa al $0.0 \%$ teñidos con Gel Red en una cámara Maxicell Primo EC340 Electroforesis Gel System; su concentración se determinó mediante curva de dilución con el ADN del bacteriófago Lambda (20 ng/ $\mu \mathrm{l})$. El ADN cuantificado se diluyó en agua tipo HPLC a un volumen total de $100 \mu \mathrm{l}$ a $10 \mathrm{ng} / \mu \mathrm{l}$ y se almacenó a $-20{ }^{\circ} \mathrm{C}$. Para la caracterización molecular se utilizaron siete cebadores RAMs sintetizados por Technologies Inc. (Tabla 2). Para la reacción de amplificación con RAMs se preparó el cóctel en un tubo estéril de microcentrífuga $(1,5 \mathrm{ml})$ para un volumen final 
Tabla 1. Procedencia de los materiales genéticos de Ibias (O. tuberosa) y Cubios (T. tuberosum) utilizados para la caracterización molecular.

\begin{tabular}{|c|c|c|c|c|}
\hline $\mathbf{N}^{\circ}$ & Procedencia & $\begin{array}{c}\text { Altura } \\
\text { (msnm) }\end{array}$ & $\begin{array}{c}\text { Lat. Norte } \\
\text { (Grados) }\end{array}$ & $\begin{array}{c}\text { Lon. Oeste } \\
\text { (Grados) }\end{array}$ \\
\hline $1 \mathrm{I}$ & Soracá & 2799 & 5,50 & 73,33 \\
\hline 21 & Ventaquemada & 2630 & 5,36 & 73,52 \\
\hline 31 & Tuta & 2600 & 5,68 & $73,22^{\circ}$ \\
\hline 41 & Combita & 2820 & 5,63 & 73,32 \\
\hline 51 & Soracá & 2799 & 5,50 & 73,33 \\
\hline 61 & Ventaquemada & 2630 & 5,36 & 73,52 \\
\hline 71 & Tuta & 2600 & 5,68 & 73,22 \\
\hline 81 & Combita & 2820 & 5,63 & 73,32 \\
\hline 91 & Ventaquemada & 2630 & 5,36 & 73,52 \\
\hline 101 & Combita & 2820 & 5,63 & 73,32 \\
\hline $1 \mathrm{C}$ & Ventaquemada & 2630 & 5,36 & 73,33 \\
\hline 2C & S. P de Iguaque & 2480 & 5,61 & 73,52 \\
\hline $3 C$ & S.P de Iguaque & 2536 & 5,57 & 73,52 \\
\hline $4 C$ & Puente de Boyacá & 2820 & 5,45 & 73,48 \\
\hline $5 C$ & Ventaquemada & 2630 & 5,36 & 73,21 \\
\hline $6 C$ & Ventaquemada & 2630 & 5,36 & 73,46 \\
\hline $7 \mathrm{C}$ & S.P de Iguaque & 2536 & 5,57 & 73,52 \\
\hline $8 C$ & Puente de Boyacá & 2820 & 5,45 & 73,21 \\
\hline $9 \mathrm{C}$ & Ventaquemada & 2630 & 5,36 & 73,52 \\
\hline $10 C$ & Ventaquemada & 2630 & 5,36 & 73,52 \\
\hline $11 C$ & S.P de Iguaque & 2536 & 5,57 & 73,21 \\
\hline
\end{tabular}

de $25 \mu \mathrm{l}$. La mezcla de reacción se preparó con buffer 1X, MgCl2 1.5 mM, DNTPs 0.2 mM, Taq Polimerasa $1 \mathrm{U}$, cebador $2 \mu \mathrm{M}$ y ADN genómico 10ng.

Tabla 2. Cebadores RAMs utilizados en la caracterización molecular de los materiales de ibias (O. tuberosa) y cubios (T. tuberosum).

\begin{tabular}{ll}
\hline Cebadores & Secuencia (5'a 3') \\
\hline CCA & DDB(CCA $) 5$ \\
CGA & DHB(CGA)5 \\
ACA & BDB(ACA) 5 \\
AG & HBH(AG)7A \\
CT & DYD $(\mathrm{CT}) 7 \mathrm{C}$ \\
TG & HVH(TG)7T \\
CA & DBDA $(\mathrm{CA}) 7$ \\
\hline
\end{tabular}

Las siguientes designaciones son usadas para los sitios degenerados: H (A ó T ó C); B (G ó T ó C); V (G ó A ó C) y D (G ó A ó T).
La amplificación se llevó a cabo en un termociclador PTC 100 Programmable Termal Controller (MJ. Research, Inc.). La desnaturalización inicial fue a $95{ }^{\circ} \mathrm{C}$ durante 5 minutos; desnaturalización a $95{ }^{\circ} \mathrm{C}$ por 30 segundos, hibridación a una temperatura de $50{ }^{\circ} \mathrm{C}$ (cebador AG y CA), $55^{\circ} \mathrm{C}$ (cebador CCA-TG-CT) y $58{ }^{\circ} \mathrm{C}$ (cebador CGA) durante 45 segundos, una extensión de $72{ }^{\circ} \mathrm{C}$ por 2 minutos, 37 ciclos desde la desnaturalización a extensión y por último una extensión a $72{ }^{\circ} \mathrm{C}$ durante 7 minutos. Los productos de amplificación se separaron por electroforesis en geles de agarosa de alta resolución al 1.5\% a 90 voltios durante 3 horas visualizándose en un transiluminador.

\section{Análisis de la información}

Se generó una matriz binaria de presencia (uno) y ausencia (cero) y se utilizó el índice de similitud de Nei y Li (1979) para establecer las distancias genéticas y el método UPGMA para generar el dendrograma con el paquete estadístico NTSYS versión 2.02 PC. Para determinar los parámetros de diversidad genética se usó el programa TFPGA versión 1.3, 1997. Se determinó el $F$ estadístico insesgado con un intervalo de confianza del $95 \%$ y se realizó el Análisis de Varianza Molecular (AMOVA) con el programa GEnAlex 6.5.

\section{RESULTADOS Y DISCUSIÓN}

El análisis mediante el coeficiente de Nei$\mathrm{Li}$ a un nivel de similitud de 0.55 permitió diferenciar los materiales en dos grandes grupos (Figura 1). En el grupo I se encuentran todos los materiales de cubios (T. tuberosum) procedentes de los diferentes municipios evaluados; los agrupamientos se presentan de acuerdo con las características morfológicas de los cultivares. Hay una variación intraespecífica en unos genotipos $1 \mathrm{C}, 2 \mathrm{C}$ y $4 \mathrm{C}$ que presentan un menor valor de similitud en comparación con el resto de cultivares evaluados, ya que presentan variaciones morfológicas importantes 


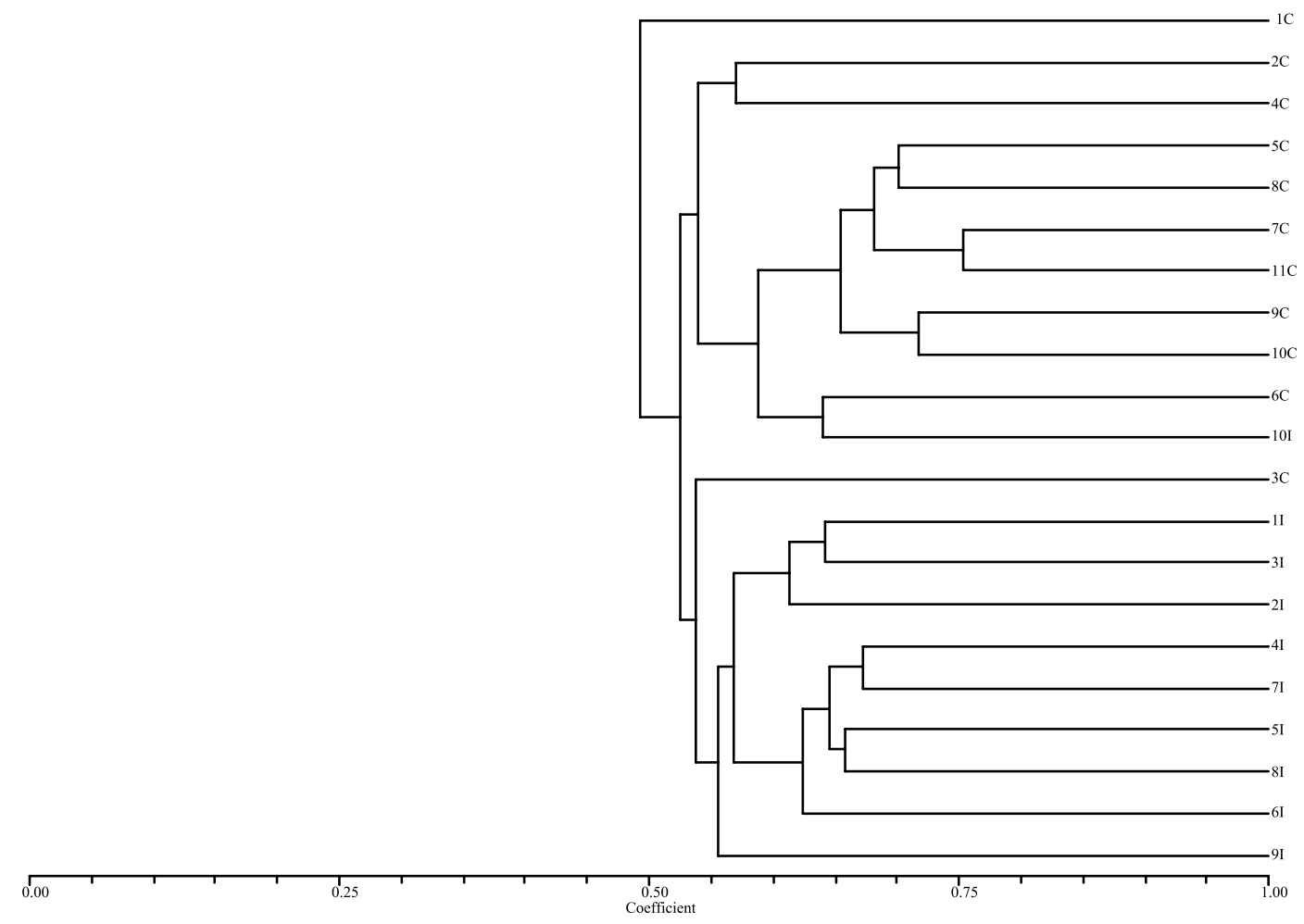

Figura 1. Dendrograma de Ibias (O. tuberosa) y de Cubios (T. tuberosum), basado en el coeficiente de similitud de Nei-Li y calculado con siete marcadores RAMs, con el método de clasificación UPGMA, SAHN y TREE de NTSYs-pc Versión 1.8 [Exeter Software, Y, USA].

en el color, la forma y el tamaño del tubérculo así como también en el color de la pulpa y en ancho y la longitud de la lámina foliar (Leguizamo 2015).

También se pudo observar que en este grupo no solo se encontraron materiales de cubios sino que el genotipo $10 \mathrm{l}$ de las ibias el cual se encuentra a un nivel de similitud de 0.60 , lo cual evidencia las relaciones intergenéricas que pueden existir entre los genotipos de Oxalis y Tropaeolum. Además presentan características morfológicas similares relacionadas con la pigmentación de las axilas, la forma del tubérculo y la longitud de la lámina foliar (Leguizamo 2015). Las distancias genéticas encontradas entre los genotipos de cubios evaluados fueron menores a las reportadas en otros estudios de diversidad genética en tubérculos andinos (Malice et al. 2010), lo cual muestra la necesidad de incrementar la variabilidad genética existente; pues el uso de una estrecha variabilidad genética en los campos de productores representa un riesgo frente a los problemas fitosanitarios.

En el grupo II se encuentran los materiales de ibias (O. tuberosa) con un valor de similaridad de 0,70 , en donde se presenta una distribución de acuerdo a características morfológicas como el color y forma del tubérculo, longitud y ancho de la lámina foliar, color de la pulpa; se puede observar también que los cultivares que presentaron menores valores de similitud fueron los procedentes del municipio de Combita, esto puede deberse a que estos presentan características morfológicas altamente diferenciables en cuanto a la forma de la hoja, color de la flor y tubérculo (Leguizamo 2015). 
En términos generales los agrupamientos de los materiales de ibias y cubios evaluados con los marcadores RAMs no se corresponden con los sitios geográficos donde estos fueron colectados y se relacionan más con características morfológicas y la especie; sin embargo se puede observar flujo genético entre las dos especies a un nivel de similitud de 0,53 lo cual puede deberse al tipo reproducción que presentan las especies y a los arreglos espaciales que utilizan los agricultores en campo. Estos resultados pueden ser tenidos en cuenta a la hora de plantear estrategias de mejoramiento genético encaminadas al incremento de la variabilidad genética en estos cultivos (Pissard et al. 2008 a).

El análisis RAMs intergenérico para las dos especies, generó un total de 127 bandas, 25 para el cebador CGA y 40 para el TG con pesos moleculares entre 300 y 1,000 Kb; el número de bandas se considera adecuado para la estimación de los parámetros genéticos (Veramendi et al. 2013; Moscoe et al. 2016). El cebador CGA fue el que mayor aporte hizo a la variación genética encontrada con un Fst de 0,39 , lo cual significa que es un marcador que debe ser tenido en cuenta a la hora de evaluar la diversidad genética en raíces y tubérculos andinos (Tabla 3).

Los valores de heterocigosidad promedio estimada variaron entre 0,37 y 0,43 para los cebadores CGA, AG y CA, respectivamente. El

Tabla 3. Parámetros genéticos obtenidos en la evaluación de la diversidad genética de cubios e ibias usando marcadores RAMs.

\begin{tabular}{lcccc}
\hline Cebador & N $^{\circ}$ Loci & He Estimada & Fst & SD \\
\hline ACA & 31 & 0,42 & 0,19 & 0,02 \\
AG & 32 & 0,43 & 0,03 & 0,01 \\
CA & 22 & 0,43 & 0,01 & 0,02 \\
CCA & 37 & 0,40 & 0,31 & 0,03 \\
CT & 33 & 0,40 & 0,03 & 0,01 \\
TG & 40 & 0,40 & 0,07 & 0,02 \\
CGA & 25 & 0,37 & 0,39 & 0,03 \\
TOTAL & $\mathbf{2 1 7}$ & $\mathbf{0 , 4 1}$ & $\mathbf{0 , 1 5}$ & $\mathbf{0 , 0 3}$ \\
\hline
\end{tabular}

valor promedio para la población total fue de 0,41 con una desviación estándar de 0,03 (Tabla 3 ), que en comparación con otros estudios de diversidad genética en otro tubérculo andino más conocido, como lo es la papa este valor es bajo (Ponce 2013). Sin embargo es más alto que lo reportado por Navarro et al. (2010) al evaluar morfoagronómica y molecularmente materiales genéticos de Solanum tuberosum L., y Solanum phureja procedentes del departamento de Nariño, con cinco marcadores RAMs $(\mathrm{He}=0.23)$. En comparación con estudios de evaluación de germoplasma de tubérculos y raíces andinas a nivel internacional los valores encontrados en este estudio son más altos (Pissard et al. 2008 b, c; Malice et al. 2010), debido principalmente a las características propias de los materiales evaluados además de que se hizo un análisis intergenérico.

Los resultados obtenidos evidencian que a pesar de la propagación vegetativa que sufren estos materiales ciclo tras ciclo la variabilidad genética persiste y se manifiesta en la diversidad de formas, tamaños, colores y texturas de sus tubérculos, esto ya había reportado anteriormente en estudios de diversidad genética en otro germoplasma (Pissard et al. 2008 a; Malice et al. 2010). Esto se corrobora con el valor de Fst que fue de 0,15 que según Wright (1978) muestra una gran diferenciación genética, lo cual es debido a la dinámica espacio-temporal que sufren estas especies en su entorno natural, los procesos de selección, domesticación y reproducción vegetativa que sufren ciclo tras ciclo. El Análisis de Varianza Molecular (AMOVA) muestra que el $87 \%$ de la variación total observada en los materiales de cubios e ibias evaluadas se encuentra dentro de cada uno de los grupos y que el $13 \%$ restante se debe al componente de la varianza genética entre grupos (Tabla 4). Por tanto, existe una mayor variación a nivel intraespecífico que muestra que deben ser considerados niveles de subdivisión y jerarquización mayor a los de este estudio. Sin embargo, el valor de varianza 
Tabla 4. Análisis de Varianza Molecular para los grupos formados con los materiales de ibias (O. tuberosa) y cubios (T. tuberosum) evaluados con los marcadores RAMs.

\begin{tabular}{lccccc}
\hline $\begin{array}{c}\text { Fuente de } \\
\text { Variación }\end{array}$ & $\begin{array}{c}\text { Grados de } \\
\text { libertad }\end{array}$ & $\begin{array}{c}\text { Suma de } \\
\text { Cuadrados }\end{array}$ & $\begin{array}{c}\text { Cuadrado } \\
\text { Medio }\end{array}$ & $\begin{array}{c}\text { Varianza } \\
\text { Estimada }\end{array}$ & Porcentaje \\
\hline Entre Grupos & 1 & 120,50 & 120,50 & 6,99 & 13 \\
Dentro de grupos & 19 & 897,78 & 47,25 & 47,25 & 87 \\
Total & $\mathbf{2 0}$ & $\mathbf{1 0 1 8 , 2 9}$ & & $\mathbf{5 4 , 2 4}$ & $\mathbf{1 0 0}$ \\
\hline
\end{tabular}

entre grupos es significativo y debe ser utilizado en las estrategias de manejo y conservación de estos recursos genéticos. Resultados similares han sido reportados en tubérculos y raíces andinas (Malice et al. 2007, 2010; Onamu et al. 2015).

La estimación de los diferentes parámetros genéticos en estos dos tubérculos andinos muestra la existencia de variabilidad genética la cual puede deberse al arreglo espacial que utilizan los agricultores en sus parcelas cultivando muchas variedades de tubérculos andinos simultáneamente en cada ciclo, y que ellos mismos hacen una selección de los mejores materiales especialmente por características relacionadas con el tubérculo. Otro factor que influencia la diversidad genética de estos cultivos es el constante intercambio de material de siembra entre los agricultores de las diferentes zonas productoras (Malice et al. 2007, 2010; Moscoe et al. 2016).

Se encontraron valores de similitud de 0,65 entre los materiales de cubios e ibias evaluados lo que pone manifiesto la existencia de flujo genético entre estas dos especies el cual se ve favorecido como se mencionaba anteriormente por el arreglo espacial en campo, las formas de reproducción que favorecen la polinización cruzada, debido a la presencia de autoincompatibilidad en ibias y producción de semillas sexuales de alta viabilidad, el nivel de ploídia, entre otros (Arbizu y Tapia 1992; Pissard et al. 2006). Los anteriores factores deben ser tenidos en cuenta para la producción de híbridos interespecíficos e intergenéricos en raíces y tubérculos andinos los cuales ya han sido generados en otras especies como una alternativa para incrementar la variabilidad genética (Cara et al. 2013; Wang et al. 2013; Cui et al, 2015; Wen et al. 2016).

La hibridación y recombinación es un evento importante en la evolución de las especies de papa y varios autores sugieren que más del 10\% de las especies de Solanum sección Petota son el resultado de hibridación (Cara et al. 2013). A través de esta se genera un nuevo patrón epigenético que es rápidamente establecido y mantenido en las poblaciones naturales, lo que permite una mayor adaptabilidad de los híbridos a los diferentes ambientes. La variación epigenética y genética juegan un papel importante en las relaciones evolutivas, sistemáticas y filogenéticas de las especies que pueden ser estudiados a través de procesos de hibridación interespecífica o intergenérica (Cara et al. 2013).

\section{CONCLUSIONES}

El análisis mediante la técnica RAMs permitió determinar la diversidad genética existente en los materiales de cubios e ibias del departamento de Boyacá, se identificó la existencia de una alta variación a nivel intraespecífico lo cual sugiere niveles de subdivisión mayores a los considerados en este estudio.

Los parámetros genéticos evidenciaron similitud genética entre estas dos especies de tubérculos andinos, poniendo de manifiesto flujo genético que puede ser aprovechado en esquemas de 
hibridación tendientes a la generación de materiales genéticos con las características agronómicas y de mercado deseadas por los agricultores, productores y consumidores.

\section{REFERENCIAS}

Aguirre, S., Piraneque, N. y Pérez, I. 2012. Sistema de producción de tubérculos andinos en Boyacá, Colombia. Cuadernos de Desarrollo Rural 9 (69): 257-273.

Arbizu, C. y Tapia, M. 1992. Tubérculos andinos. En: J. Hernández y J. León. (eds.). Cultivos marginados: otra perspectiva de 1492. FAO, Roma, p. 147-161.

Arias, C., e Higuera, M. 2011. Análisis y comparación de los glucosinolatos presentes en diferentes accesiones de cubio (Tropaeolum tuberosum) para evaluar su uso potencial en el control del patógeno de la papa Spongospora subterránea. Tesis de maestría, Universidad Nacional de Colombia, Bogotá.

Bradbury, E. and Emshwiller, E. 2011. The role of organic acids in the domestication of Oxalis tuberosa: a new model for studying domestication resulting in opposing crop phenotypes. Economic Botany 65(1): 7684.

Cara, N., Marfil, C. and Masuelli, R. 2013. Epigenetic patterns newly established after interspecific hybridization in natural populations of Solanum. Ecology and Evolution 3(11): 3764-3779.

Chirinos, R., Campos, D., Costa, N., Arbizu, C., Pedreschi, R. and Larondelle Y. 2008 a. Phenolic profiles of Andean mashua (Tropaeolum tuberosum Ruíz and Pavón) tubers: Identification by HPLC-DAD and evaluation of their antioxidant activity. Food Chemistry 106(3): 1285-1298.
Chirinos, R., Campos, D., Warnier, M., Pedreschi, R., Rees, J. and Larondelle, Y. 2008 b. Antioxidant properties of mashua (Tropaeolum tuberosum) phenolic extracts against oxidative damage using biological in vitro assays. Food Chemistry 111 (1): 98-105.

Chirinos, R., Rogez, H., Campos, D., Pedreschi, R. and Larondelle, Y. 2007. Optimization of extraction conditions of antioxidant phenolic compounds from mashua (Tropaeolum tuberosum Ruíz y Pavón) tubers. Separation and Purification Technology 55(2): 217-225.

Cui, H., Sun, Y., Deng, J. and Xia, G. 2015. Chromosome elimination and introgression following somatic hybridization between bread wheat and other grass species. Plant Cell Tissue and Organ Culture 120(1): 203-210.

Dellaporta, S., Wood, J. and Hicks, J. 1983. A plant DNA minipreparation: Versión II. Plant Molecular Biology Reporter 14(1):19-21.

Emshwiller, E., Theim, T., Grau, A., Nina, V. and Terrazas, F. 2009. Origins of domestication and polyploidy in oca (Oxalis tuberosa; Oxalidaceae). 3. AFLP data of oca and four wild, tuber-bearing taxa. American Journal of Botany 96(10): 1839-1848.

Hershkovitz, M., Hernández, C. and Arroyo, M. 2006. Ribosomal DNA evidence for the diversification of Tropaeolum sect. Chilensia (Tropaeolaceae). Plant Systematics and Evolution 260(1): 1-24.

Jiménez, M., Rossi, A. and Sammán N. 2015. Health properties of oca (Oxalis tuberosa) and yacon (Smallanthus sonchifolius). Food Functions 6(10): 3266-3271. 
Leguizamo, M. 2015. Colecta, caracterización morfológica y molecular de tubérculos andinos ibias (Oxalis tuberosa) y cubios (Tropaeolum tuberosum) en el departamento de Boyacá. Tesis de pregrado, Universidad Pedagógica y Tecnológica de Colombia, Tunja.

Malice, M. 2009. Genetic diversity and structure of three Andean tubers: Oxalis tuberosa Molina, Ullucus tuberosus Caldas and Tropaeolum tuberosum Ruiz and Pav. Thesis of PhD. Agricultural University, Belgium.

Malice, M., Bizoux, J., Blas, R. and Baudoin J. 2010. Genetic diversity of Andean tuber crop species in the in situ microcenter of Huanuco, Perú Crop Science 50(5): 1915-1923.

Malice, M., Martin, N., Pissard, A., Rojas, J., Gandarillas, A., Bertin, P. and Baudoin, J. 2007. A preliminary study of the genetic diversity of Bolivian oca (Oxalis tuberosa Mol.) varieties maintained in situ and ex situ through the utilization of ISSR molecular markers. Genetic Resources Crop Evolution 54(4): 685-690.

Manrique, I., Arbizu, C., Vivanci, F., González, R., Ramírez, C., Chávez, O., Tay, D. y Elís, D. 2013. Tropaeolum tuberosum Ruíz and Pav. Colección de germoplasma de mashua conservada en el Centro Internacional de la Papa (CIP). Primera Edición. Centro Internacional de la Papa, La Molina, Perú, p. 34-77.

Marfil, C., Masuelli, R., Davison, J. and Comai, L. 2006. Genomic instability in Solanum tuberosum, Solanum kurtzianum interspecific hybrids. Genome 49(2):104113.

Monteros, A., Mazo, N. y Castillo, R. 1997. Estudio de la variación morfológica e isoenzimática de 78 entradas de mashua (Tropaeolum tuberosum) en Santa Catalina, INIAP. Quito, Ecuador, p. 34.

Morillo, A., Morillo, Y. and Pinzón H. 2015. Molecular characterization of the plum collection (Prunus domestica L. Borkh) of the Pedagogical and Technological University of Colombia. African Journal of Biotechnology 14(3): 257-263.

Moscoe, L., Blas, R., Huamán, D., Modesto, $M$. and Emshwiller, E. 2016. Genetic basis for folk classification of oca (Oxalis tuberosa Molina; Oxalidaceae): implications for research and conservation of clonally propagated crops. Genetic Resources and Crop Evolution 63(1): 1-21.

Navarro, C., Bolaños, L. y Lagos, T. 2010. Caracterización morfoagronómica y molecular de 19 genotipos de papa guata y chaucha (Solanum tuberosum L. y Solanum phureja Juz et Buk) cultivados en el departamento de Nariño. Revista de Agronomía 27(1): 27-39.

Nei, M. and Li, W. 1979. Mathematical model for studying genetic variation in terms of restriction endonuclease. Proceedings of the National Academy Sciencies 79(10): $5267-5273$.

Onamu, R., Solano, P., Castellano, J., Rodríguez, J. y Pérez, N. 2015. Diversidad genética entre variedades de papa (Solanum tuberosum L.) cultivadas en México. Revista Fitotecnia Mexicana 38(1): 7-15.

Piñeros, N. y Ñústez, I. 2004. Evaluación de la variabilidad genética de la colección de cubio (Tropaeolum tuberosum Ruíz and Pavón) reporte de investigación, Universidad Nacional de Colombia, Bogotá, p.15. 
Pissard, A., Arbizu, C., Ghislain, M. and Bertin, P. 2008a. Influence of the geographical provenance on the genetic structure and diversity of the vegetatively propagated Andean tuber crop, mashua (Tropaeolum tuberosum), highlighted by ISSR markers and multivariate analysis methods. International Journal. Plant Sciences 169(9):1248-1260.

Pissard, A., Arbizu, C., Ghislain, M., Faux, A., Paulet, S. and Bertin, D. 2008b. Congruence between morphological and molecular markers inferred from the analysis of the intramorphotype genetic diversity and the spatial structure of Oxalis tuberosa Molecular Genetics 132(1):71-85.

Pissard, A., Rojas, J., Faux, A., Paulet, S. and Bertin, P. 2008c. Evidence of intra-varietal genetic variability in the vegetatively propagated crop oca (Oxalis tuberosa Mol.) in the Andean traditional farming system. Plant Systematics and Evolution 270(1-2):59-74.

Pissard, A., Ghislain, M. and Bertin, P. 2006. Genetic diversity of the Andean tuberbearing species, oca [Oxalis tuberosa Mol.], investigated by inter-simple sequence repeats. Genome 49(1):8-16.

Ponce, R. 2013. Caracterización molecular de las variedades de papas cultivadas (Solanum spp.) más importantes del Perú mediante el uso de microsatélites. Tesis de pregrado, Universidad Nacional Mayor de San Marcos, Lima, Perú.

Rapp, R. and Wendel, J. 2005. Epigenetics and plant evolution. New Phytopathology 168(1):81-91.
Rivera, G. 2010. Elaboración y valoración nutricional de tres productos alternativos a base de mashua para escolares del proyecto Runa Kawsay. Tesis de Pregrado. Escuela Superior Politécnica de Chimborazo, Ecuador.

Rosero, M. 2010. Colección, caracterización y conservación de variabilidad genética de oca (Oxalis tuberosa Mol.) en agroecosistemas paramunos del departamento de Nariño-Colombia. Tesis de Maestría, Universidad Nacional de Colombia, Palmira.

Veramendi, S., Cadima, X. and Gabriel, J. 2013. Integración molecular y morfológica para la formación de la Colección Núcleo de papa de Bolivia. Revista Latinoamericana de la Papa 17 (2): 23-39.

Wang, J., Jiang, J. and Wang, Y. 2013. Protoplast fusion for crop improvement and breeding in China. Plant Cell Tissue and Organ Culture 112(2): 131-142.

Wen, L., Shih, H., Weng, I., Zhu, Ya., Tsai, C., Chou, C. and Chiang, Y. 2016. Characterization of Genomic Inheritance of Intergeneric Hybrids between Ascocenda and Phalaenopsis Cultivars by GISH, PCR-RFLP and RFLP. PLoS ONE 11(4): 1-14.

Wright, S. 1978. Evolution and the genetics of populations, variability within and among natural populations, Vol 4. University of Chicago Press. Chicago, p. 144. 